\title{
Electric mobility in Japan - an European perspective
}

Hartmut Stiller, business consultant e-mobility

This manuscript was not available on completion of this publication.

Thank you for your understanding. 